\title{
Stabilization of Parametric Roll Resonance with Active U-Tanks via Lyapunov Control Design
}

\author{
Christian Holden*, Roberto Galeazzi ${ }^{\dagger}$, Thor I. Fossen*, Tristan Perez ${ }^{\ddagger}$
}

\begin{abstract}
Parametric ship roll resonance is a phenomenon where a ship can rapidly develop high roll motion while sailing in longitudinal waves. This effect can be described mathematically by periodic changes of the parameters of the equations of motion, which lead to a bifurcation. In this paper, the control design of an active u-tank stabilizer is carried out using Lyapunov theory. A nonlinear backstepping controller is developed to provide global exponential stability of roll. An extension of commonly used u-tank models is presented to account for large roll angles, and the control design is tested via simulation on a high-fidelity model of a vessel under parametric roll resonance.
\end{abstract}

\section{INTRODUCTION}

Parametric resonance is a phenomenon that can be explained in terms of time-dependent changes in the parameters of the governing dynamic equations [1]. For some ships, parametric resonance can occur in roll - rotation about the longitudinal axis. The resulting heavy roll motion, which can reach 30-40 degrees of roll angle, may bring the vessel into dangerous conditions that can even result in capsize. Container ships and fishing vessels are known to be prone to parametric roll, and several incidents have been reported with significant damage to cargo as well as structural damages for millions of dollars [2], [3].

The cause of this unstable roll motion in ships is the quasi-periodic variation of the hydrostatic forces due to the passage of the waves. This variation is due to a change in the hull submerged volume. Hydrostatic forces can be described in terms of a non-linear parametric model, and the changes in the hull submerged volume results in periodic variations of the parameters of such model. Under the following (empirical) conditions, parametric roll resonance will then develop:

- The vessel is sailing against the waves (head seas) or with the waves (following seas).

- The length of the waves is close to the length of the vessel $\left(\lambda_{w} \approx L_{\mathrm{PP}}\right)$.

- The frequency of the waves as observed from ship (the "encounter frequency") is close to twice the natural roll frequency $\left(\omega_{e} \approx 2 \omega_{0}\right)$.

- The roll damping is sufficiently low.

- The vessel must have a specific hull shape (e.g. pronounced bow flare).

* Dept. of Engineering Cybernetics, Norwegian University of Science and Technology c.holden@ieee. org, fossen@ieee.org

${ }^{\dagger}$ DTU Electrical Engineering, Technical University of Denmark rgdelektro.dtu.dk

$\ddagger$ ARC Centre for Complex Dynamic Systems and Control, The University of Newcastle, Australia tristan.perez@newcastle.edu.au
To prevent the development of parametric resonance, one or more of the above conditions can to be avoided. For example, [4] proposed detuning the resonance condition (making $\omega_{e} \neq 2 \omega_{0}$ ) by speed change combined with increase of damping via active control of fin stabilizers.

Many ships are equipped with u-tanks as a roll stabilization device. A u-tank consists of two large reservoirs on the port and starboard side of the vessel, connected by a duct at the bottom to let water pass from one reservoir to the other. As the vessel rolls, the fluid in the reservoirs move out of phase with the roll, and thus the weight of the fluid provides a roll moment that opposes that induced by the waves. In passive u-tanks, the water can flow freely. In active u-tanks, a pump can be used to force the fluid from one side to the other to adapt to the prevailing sea conditions. A passive model for use with parametric roll was presented in [6]. It was capable of reducing susceptibility to parametric roll, but not stopping it if it occurred.

In this paper, we consider an active tank and design the controller using Lyapunov theory. A nonlinear backstepping controller is developed to provide global exponential stability of roll, thus eliminating the problem entirely. An extension commonly used u-tank models (see e.g. [7], [8]) is presented to account for large roll angles, and the control design is tested via simulation on the high-fidelity model of a vessel under roll parametric resonance of [9].

\section{MODEL}

\section{A. Modeling Hypothesis}

In order to ease the design work, some important assumptions are made in modeling parametric roll resonance:

1) The ship is sailing in head seas.

2) The ship is subjected to regular, sinusoidal waves.

3) The ship's motion in surge, sway and yaw can be neglected.

In addition, some assumptions are made regarding the u-tank:

4) The liquid surface in the u-tank is flat.

5) The liquid is incompressible.

6) The air in the u-tank is an ideal gas.

7) The velocity of the fluid is constant in space (but not time) apart from at the duct/reservoir junctions.

\section{B. Parametric Roll Model}

There are many models of parametric roll of varying degree of complexity. One of the most advanced ones can be found in [9]. It includes couplings between heave, roll and pitch, and uses a third-order Taylor approximation to these 
couplings. Defining the state $\chi=[z, \phi, \theta]^{\mathrm{T}}$ as the vector of heave, roll and pitch, the model can be written as

$$
(\mathcal{M}+\mathcal{A}) \ddot{\chi}+\mathcal{D}(\dot{\phi}) \dot{\chi}+c_{\text {res }}(\chi, t)=c_{\text {ext }}(t),
$$

where

$$
\begin{aligned}
M & =\operatorname{diag}\left(\left[m, I_{x}, I_{y}\right]\right)>0 \\
A & =-\left[\begin{array}{ccc}
Z_{\ddot{z}} & 0 & Z_{\ddot{\theta}} \\
0 & K_{\ddot{\phi}} & 0 \\
M_{\ddot{z}} & 0 & M_{\ddot{\theta}}
\end{array}\right]>0 \\
D(\dot{\phi}) & =-\left[\begin{array}{ccc}
Z_{\dot{z}} & 0 & Z_{\dot{\theta}} \\
0 & -\bar{K}_{\dot{\phi}}(\dot{\phi}) & 0 \\
M_{\dot{z}} & 0 & M_{\dot{\theta}}
\end{array}\right]>0 \\
\bar{K}_{\dot{\phi}}(\dot{\phi}) & =K_{\dot{\phi}}+K_{|\dot{\phi}| \dot{\phi}}|\dot{\phi}|>0 \\
c_{\mathrm{ext}}(t) & =\left[\bar{Z}_{\mathrm{ext}} \cos \left(\omega_{e} t+\alpha_{3}\right), 0, \bar{M}_{\mathrm{ext}} \cos \left(\omega_{e} t+\alpha_{5}\right)\right]^{\mathrm{T}} .
\end{aligned}
$$

The term $c_{\text {res }}(\chi, t)$ is the nonlinear restoring force couplings between heave, roll, pitch and the wave motion (the latter an explicit function of time).

Further details of this model - including coefficients calculated for a specific $281 \mathrm{~m}, 76500$ tonne container ship - can be found in [9].

This model is very good at capturing the dynamics of parametric roll, but is unwieldy for control design. A much simpler model, that still does an adequate job of describing the dynamics of the system, is

$$
\left(I_{x x}-K_{\ddot{\phi}}\right) \ddot{\phi}+\bar{K}_{\dot{\phi}}(\dot{\phi}) \dot{\phi}+g m \overline{G M}(t) \phi+K_{\phi^{3}} \phi^{3}=0,
$$

where $I_{x x}$ is the rigid-body moment of inertia about the body-fixed $x$ axis, $K_{\ddot{\phi}}<0$ is the hydrodynamic added mass, $\bar{K}_{\dot{\phi}}(\dot{\phi})=K_{\dot{\phi}}+K_{|\dot{\phi}| \dot{\phi}}|\dot{\phi}|>0$ is nonlinear damping, $g$ the acceleration of gravity, $m$ the mass of the vessel, $K_{\phi^{3}}>0$ a nonlinear spring term, and the metacentric height $\overline{G M}(t)$ is given as

$$
\overline{G M}(t)=\overline{G M}_{0}+\overline{G M}_{t} \cos \omega_{e} t
$$

where $\omega_{e}$ is the encounter frequency [10].

The parameters of the high-fidelity model (1) (henceforth referred to as the plant model) were derived in [9]. The parameters of the simplified model (2) (henceforth referred to as the nominal model) can be derived from the parameters of (1).

The nominal model was be used to design the controller, while the plant model was used for verification.

\section{U-Tank Model}

In [7] a linear model of a passive u-tank is developed under the assumption of small roll angles and low height difference between the fluid level in the port and starboard side reservoirs. For the purpose of controlling parametric roll, the model must take into account the possibility of highamplitude roll, and the u-tank must be active. In addition, in the forward direction, the length of the duct may be different from the length of the reservoirs. The model of [7] is therefore expanded in this section.
A u-tank design as in Fig. 1 is used. It has dimensions as given in the figure, and two pumps; one in the fluid duct at the bottom of the u-tank, and one a the air duct at the top of the u-tank. The pump in the fluid duct is assumed to generate the pressure $P_{w}$ and the pump in the air duct the differential air pressure $P_{a}$.

The notation used is defined in Fig. 1. $C_{f}$ is the ship centre of flotation, around which the ship rolls. The state variable $\xi$ is the height difference between the fluid levels in the port and starboard reservoirs. The reservoirs and the duct have constant cross-sectional areas $A_{r}$ and $A_{d}$ taken perpendicular to the $y$-axis. The roll angle $\phi$ is positive to starboard.

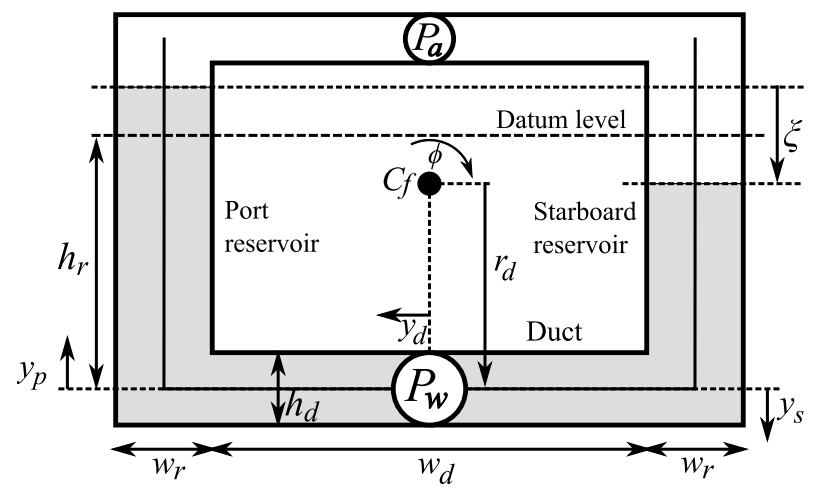

Fig. 1. u-tank notation

The spatial dimension $y$ runs along the midsection of the tank, giving the three axes $y_{s}, y_{d}$ and $y_{p}$. It is assumed that there is no flow in any direction perpendicular to the $y$ axes. The motions of the fluid in the tank will then be governed by a simplified version of Euler's Equation

$$
\rho_{t} \frac{\partial v}{\partial t}+\rho_{t} v \frac{\partial v}{\partial y}=\rho_{t} Y-\frac{\partial P}{\partial y}
$$

if the fluid has density $\rho_{t}$, where $Y$ is the external force per unit of mass [7].

Assuming that the motion at the junctions can be neglected, Euler's Equation reduces to

$$
\rho_{t} \frac{\mathrm{d} v}{\mathrm{~d} t}=\rho_{t} Y-\frac{\mathrm{d} P}{\mathrm{~d} y} .
$$

This simplification is justified by the fact that the exact behavior of the fluid at the intersections is irrelevant; only the effect at the surface level in each reservoir is interesting for the purpose of this paper.

The velocity $v_{r}$ of the fluid in the reservoirs is given by

$$
v_{r}=\frac{\mathrm{d} \xi / 2}{\mathrm{~d} t} \triangleq \frac{\dot{\xi}}{2}
$$

and anywhere in the tank by

$$
v=\frac{A_{r} v_{r}}{n_{a}}=\frac{A_{r} \dot{\xi}}{2 n_{a}},
$$

where

$$
n_{a} \triangleq \begin{cases}A_{d} & \text { in the duct } \\ A_{r} & \text { in the reservoirs. }\end{cases}
$$


The external force per unit mass $Y$ is made up of the following factors:

1) Component of acceleration due to gravity in the $y$ direction: $-g \cos \phi_{1}$.

2) The acceleration due to roll acceleration: $-r \ddot{\phi} \sin \phi_{2}$.

3) Friction: $-q_{v} n_{a} v-q_{v^{2}} n_{a}^{2}|v| v$ where

$$
\begin{aligned}
q_{v} & = \begin{cases}q_{v, d} & \text { in the duct } \\
q_{v, r} & \text { in the reservoirs }\end{cases} \\
q_{v^{2}} & = \begin{cases}q_{v^{2}, d} & \text { in the duct } \\
q_{v^{2}, r} & \text { in the reservoirs }\end{cases}
\end{aligned}
$$

are parameters to be determined by experiment.

4) Duct pump:

$$
Y_{P}=\left\{\begin{array}{cl}
\frac{1}{\rho_{t} w} P_{w} & \text { in the duct } \\
0 & \text { in the reservoirs. }
\end{array}\right.
$$

The variables $w \triangleq w_{r}+w_{d}, \phi_{1}, \phi_{2}$ and $r$ are as defined in Fig. 2.

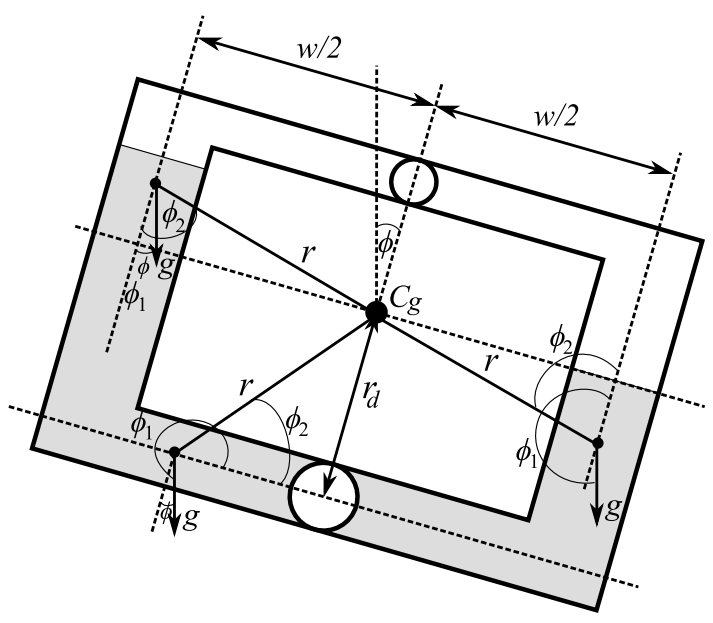

Fig. 2. External forces applied to fluid in the tank

Inserting the above into (4), we get

$$
\begin{gathered}
\frac{\rho_{t} A_{r}}{2 n_{a}} \ddot{\xi}+\frac{\rho_{t} q_{v} A_{r}}{2} \dot{\xi}+\frac{\rho_{t} q_{|v| v} A_{r}^{2}}{4}|\dot{\xi}| \dot{\xi}+\rho_{t} g \cos \phi_{1} \\
+\rho_{t} r \ddot{\phi} \sin \phi_{2}=\rho_{t} Y_{P}-\frac{\mathrm{d} P}{\mathrm{~d} y} .
\end{gathered}
$$

Integrating (5) over $y$ gives

$$
\begin{gathered}
\frac{\rho_{t} A_{r} I_{1}}{2} \ddot{\xi}+\frac{\rho_{t} A_{r} I_{2}}{2} \dot{\xi}+\frac{\rho_{t} A_{r}^{2} I_{3}}{4}|\dot{\xi}| \dot{\xi}+\rho_{t} g I_{4} \\
+\rho_{t} \ddot{\phi} I_{5}=P_{w}+P_{s}-P_{p},
\end{gathered}
$$

where

$$
\begin{aligned}
I_{1} & \triangleq \int_{\operatorname{tank}} \frac{\mathrm{d} y}{n_{a}}=\frac{1}{A_{d}} \int_{-w / 2}^{w / 2} \mathrm{~d} y_{d}+\frac{1}{A_{r}} \int_{-h_{r}+\xi / 2}^{0} \mathrm{~d} y_{s}+\frac{1}{A_{r}} \int_{0}^{h_{r}+\xi / 2} \mathrm{~d} y_{p} \\
& =\frac{w}{A_{d}}+\frac{2 h_{r}}{A_{r}} \\
I_{2} & \triangleq \int_{\operatorname{tank}} q_{v} \mathrm{~d} y=q_{v, d} \int_{-w / 2}^{w / 2} \mathrm{~d} y_{d}+q_{v, r} \int_{-h_{r}+\xi / 2}^{0} \mathrm{~d} y_{s}+q_{v, r} \int_{0}^{h_{r}+\xi / 2} \mathrm{~d} y_{p} \\
& =w q_{v, d}+2 h_{r} q_{v, r}
\end{aligned}
$$

$$
\begin{aligned}
& I_{3} \triangleq \int_{\operatorname{tank}} q_{v^{2}} \mathrm{~d} y=q_{v^{2}, d} \int_{-w / 2}^{w / 2} \mathrm{~d} y_{d}+q_{v^{2}, r} \int_{-h_{r}+\xi / 2}^{0} \mathrm{~d} y_{s}+q_{v^{2}, r} \int_{0}^{h_{r}+\xi / 2} \mathrm{~d} y_{p} \\
& =w q_{v^{2}, d}+2 h_{r} q_{v^{2}, d} \\
& I_{4} \triangleq \int_{\operatorname{tank}}^{\cos \phi_{1}} \mathrm{~d} y=\int_{-w / 2}^{w / 2} \sin \phi \mathrm{d} y_{d}-\int_{-h_{r}+\xi / 2}^{0} \cos \phi \mathrm{d} y_{s}+\int_{0}^{h_{r}+\xi / 2} \cos \phi \mathrm{d} y_{p} \\
& =w \sin \phi+\xi \cos \phi \\
& I_{5} \triangleq \int_{\operatorname{tank}}^{r \sin \phi_{2}} \mathrm{~d} y=r_{d} \int_{-w / 2}^{w / 2} \mathrm{~d} y_{d}+\frac{w}{2} \int_{-h_{r}+\xi / 2}^{0} \mathrm{~d} y_{s}+\frac{w}{2} \int_{0}^{h_{r}+\xi / 2} \mathrm{~d} y_{p} \\
& =w\left(r_{d}+h_{r}\right) \text {. }
\end{aligned}
$$

The pressure differential between starboard and port reservoirs $P_{s}-P_{p}$ is given by

$$
P_{s}-P_{p}=P_{a}
$$

Rewriting (6) and multiplying on both sides by $A_{r} / 2$ gives

$$
m_{\xi} \ddot{\xi}+d_{\xi}(\dot{\xi}) \dot{\xi}+k_{\xi}(\phi) \xi+f_{\phi}(\phi, \ddot{\phi})=u
$$

given in Newtons, where

$$
\begin{aligned}
m_{\xi} \triangleq & \frac{1}{2} A_{r} \rho_{t}\left(\frac{w A_{r}}{2 A_{d}}+h_{r}\right) \\
d_{\xi}(\dot{\xi}) \triangleq & \frac{1}{4} A_{r}^{2} \rho_{t}\left(w q_{v, d}+2 h_{r} q_{v, r}\right) \\
& +\frac{1}{8} A_{r}^{2} \rho_{t}\left(w q_{v^{2}, d}+2 h_{r} q_{v^{2}, d}\right)|\dot{\xi}| \\
k_{\xi}(\phi) \triangleq & \frac{1}{2} A_{r} \rho_{t} g \cos \phi \triangleq 2 \alpha_{\xi^{2}} \cos \phi \\
f_{\phi}(\phi, \ddot{\phi}) \triangleq & \frac{1}{2} A_{r} \rho_{t} g w \sin \phi+\frac{1}{2} A_{r} \rho_{t} w\left(r_{d}+h_{r}\right) \ddot{\phi} \\
u \triangleq & \frac{1}{2} A_{r}\left(P_{a}+P_{w}\right) .
\end{aligned}
$$

Note that only the parameters $q_{v}$ and $q_{v^{2}}$ need to be determined by experiment.

\section{Parametric Roll Model Redux}

As the roll motion acts on the u-tank, so does the u-tank act on the roll motion. This is predominantly caused by two factors: The weight of the tank fluid and the acceleration of the fluid (as per Newton's Second and Third Laws). According to [7], a moment $f_{\xi}$ acts on on the right-hand side of the roll motion of (1) and (2), given by

$$
\begin{aligned}
f_{\xi}(\xi, \ddot{\xi}) & =-\frac{w}{2} m_{r} \dot{v}_{r}-r_{d} m_{d} \dot{v}_{d}-\frac{w^{2}}{2} \rho_{t} g A_{r} \xi \\
& =-\frac{\rho_{t} w A_{r}\left(h_{r}+r_{d}\right)}{2} \ddot{\xi}-\frac{\rho_{t} g w A_{r}}{2} \xi \\
& \triangleq-\alpha_{\ddot{\xi}} \ddot{\xi}-\alpha_{\xi} \xi .
\end{aligned}
$$

The acceleration of the tank fluid $\ddot{\xi}$ is only relative to the tank walls (i.e. the ship). The tank walls rotate with the ship, giving an extra acceleration component proportional to the angular acceleration. Also, even with $\xi=0$, the tank fluid adds weight to the ship, giving an extra term

$-f_{g}=g \rho_{t} \int_{-w / 2}^{w / 2} A_{d} r_{d} \sin \phi \mathrm{d} y+g \rho_{t} \int_{-h_{r}+\xi / 2}^{0} \underset{r}{A_{r}}\left(r_{d}+y\right) \sin \phi \mathrm{d} y$ 


$$
\begin{aligned}
& +g \rho_{t} \int_{0}^{h_{r}+\xi / 2} A_{r}\left(r_{d}-y\right) \sin \phi \mathrm{d} y \\
= & g \rho_{t} A_{r}\left(2 r_{d} h_{r}-h_{r}^{2}+\frac{A_{d}}{A_{r}} r_{d} w\right) \sin \phi \triangleq \alpha_{t} \sin \phi .
\end{aligned}
$$

Together, this this gives a total tank-induced moment of

$$
-f_{u}(\ddot{\phi}, \ddot{\xi}, \phi, \xi)=\alpha_{\ddot{\xi}} \ddot{\xi}+\alpha_{\xi} \xi+\alpha_{t} \sin \phi+I_{t} \ddot{\phi}
$$

where $I_{t} \triangleq \int_{\operatorname{tank}} r^{2} \mathrm{~d} V$ is the moment of inertia of the fluid in the tank. $I_{t}$ is a function of $\xi$, but for simplicity it is calculated for $\xi=0$.

\section{E. Resulting Model}

1) Plant Model: Defining $\bar{\chi} \triangleq\left[\chi^{\mathrm{T}}, \xi\right]^{\mathrm{T}} \in \mathbb{R}^{4}$, the resulting tank-ship plant model becomes

$$
(\overline{\mathcal{M}}+\overline{\mathcal{A}}) \ddot{\bar{\chi}}+\overline{\mathcal{D}}(\dot{\bar{\chi}}) \dot{\bar{\chi}}+\bar{c}_{\text {res }}(\bar{\chi}, t)=\bar{c}_{\text {ext }}(\tau, t)
$$

where

$$
\begin{aligned}
& \overline{\mathcal{M}} \triangleq\left[\begin{array}{cc}
\mathcal{M}+\operatorname{diag}\left(\left[0, I_{t}, 0\right]\right) & \bar{\alpha}_{\ddot{\xi}} \\
\bar{\alpha}_{\ddot{\xi}}^{\mathrm{T}} & m_{\xi}
\end{array}\right] \in \mathbb{R}^{4 \times 4} \\
& \bar{\alpha}_{\ddot{\xi}} \triangleq\left[0, \alpha_{\ddot{\xi}}, 0\right]^{\mathrm{T}} \in \mathbb{R}^{3} \\
& \overline{\mathcal{A}} \triangleq\left[\begin{array}{cc}
\mathcal{A} & 0 \\
0 & 0
\end{array}\right] \in \mathbb{R}^{4 \times 4} \\
& \overline{\mathcal{D}}(\dot{\bar{\chi}}) \triangleq\left[\begin{array}{cc}
\mathcal{D}(\dot{\phi}) & 0 \\
0 & d_{\xi}(\dot{\xi})
\end{array}\right] \in \mathbb{R}^{4 \times 4} \\
& \bar{c}_{\text {res }}(\bar{\chi}, t) \triangleq\left[c_{\text {res }}^{\mathrm{T}}(\chi, t)+\bar{\alpha}_{\xi}^{\mathrm{T}}, k_{\xi}(\phi) \xi+\alpha_{\xi} \sin \phi\right]^{\mathrm{T}} \in \mathbb{R}^{4} \\
& \bar{\alpha}_{\xi} \triangleq\left[0, \alpha_{\xi} \xi+\alpha_{t} \sin \phi, 0\right]^{\mathrm{T}} \in \mathbb{R}^{3} \\
& \bar{c}_{\text {ext }}(u, t) \triangleq\left[c_{\text {ext }}^{\mathrm{T}}(t), u\right]^{\mathrm{T}} \in \mathbb{R}^{4} \text {. }
\end{aligned}
$$

2) Nominal Model: Defining $x_{1} \triangleq \phi, x_{2} \triangleq \xi, v_{1} \triangleq \dot{\phi}$ and $v_{2} \triangleq \dot{\xi}$, the resulting tank-ship nominal model becomes

$$
\begin{aligned}
\dot{x} & =v \\
M \dot{v}+D(v) v+k(x, t) & =B u
\end{aligned}
$$

where

$$
\begin{aligned}
& M \triangleq\left[\begin{array}{cc}
I_{x x}-K_{\ddot{\phi}}+I_{t} & \alpha_{\ddot{\xi}} \\
\alpha_{\ddot{\xi}} & m_{\xi}
\end{array}\right] \\
& D(v) \triangleq \operatorname{diag}\left(\left[\bar{K}_{\dot{\phi}}\left(v_{1}\right), d_{\xi}\left(v_{2}\right)\right]\right) \\
& k(x, t) \triangleq\left[g m \overline{G M}(t) x_{1}+K_{\phi^{3}} x_{1}^{3}+\alpha_{t} \sin x_{1}+\alpha_{\xi} x_{2},\right. \\
&\left.\quad \alpha_{\xi} \sin x_{1}+2 \alpha_{\xi^{2}} x_{2} \cos x_{1}\right]^{\mathrm{T}} \\
& B \triangleq[0,1]^{\mathrm{T}} .
\end{aligned}
$$

\section{CONTROLler}

\section{A. Control Objective}

With both the plant model and the nominal model, the output of the system is taken to be $y=[\phi, \dot{\phi}]^{\mathrm{T}}$, which should be driven to zero. The internal states (the tank states in both models, and in addition the heave and pitch motions in the nominal model) should be at least bounded, or the controller cannot work in a real-life setting.

The heave and pitch motions are known to be bounded in the conditions examined in this paper [11], and unaffected by the u-tank [7]. Thus, the control objective is to drive $y$ to zero, while keeping the tank states bounded.

\section{B. Design and Analysis}

Backstepping will be used to design the control law. It is assumed that, in addition to the assumptions in Section II-A, the states can be accurately measured.

We first consider the $x_{1}$ subsystem with $v_{1}$ considered as input. Using the control Lyapunov function $V_{1}\left(x_{1}\right) \triangleq x_{1}^{2} / 2$, we find its derivative to be given by

$$
\dot{V}_{1}=x_{1} \dot{x}_{1}=v_{1} x_{1}=-c_{1} x_{1}^{2}+x_{1} \nu_{1}
$$

with $\nu_{1} \triangleq v_{1}+c_{1} x_{1} \cdot \dot{V}_{1}$ is negative definite with $\nu_{1}=0$.

We find

$$
\begin{aligned}
\dot{x}_{1}= & -c_{1} x_{1}+\nu_{1} \\
|M| \dot{\nu}_{1}= & |M| c_{1} v_{1}-\alpha_{\ddot{\xi}} u-m_{\xi} \bar{K}_{\dot{\phi}}\left(v_{1}\right) v_{1}+\alpha_{\ddot{\xi}} d_{\xi}\left(v_{2}\right) v_{2} \\
& -m_{\xi} g m \overline{G M}(t) x_{1}+2 \alpha_{\ddot{\xi}} \alpha_{\xi^{2}} x_{2} \cos x_{1}-m_{\xi} \alpha_{\xi} x_{2} \\
& -m_{\xi} K_{\phi^{3}} x_{1}^{3}+\left(\alpha_{\ddot{\xi}} \alpha_{\xi}-m_{\xi} \alpha_{t}\right) \sin x_{1}
\end{aligned}
$$

where $|M| \triangleq \operatorname{det}(M)$.

Next we consider the $x_{1}-\nu_{1}$ subsystem, with $u$ considered as input. Using the control Lyapunov function $V_{2}\left(x_{1}, \nu_{1}\right) \triangleq$ $V_{1}\left(x_{1}\right)+|M| \nu_{1}^{2} / 2+m_{\xi} g m \overline{G M}_{0} x_{1}^{2} / 2+m_{\xi} K_{\phi^{3}} x_{1}^{4} / 4$, we find its derivative to be given by

$$
\begin{aligned}
\dot{V}_{2}= & -c_{1}\left(1+m_{\xi} g m \overline{G M}_{0}+m_{\xi} K_{\phi^{3}} x_{1}^{2}\right) x_{1}^{2}+\nu_{1}\left[x_{1}\right. \\
& +|M| c_{1} v_{1}-m_{\xi} g m \overline{G M}_{t} \cos \left(\omega_{e} t\right) x_{1}+\alpha_{\ddot{\xi}} d_{\xi}\left(v_{2}\right) v_{2} \\
& -m_{\xi} \bar{K}_{\dot{\phi}}\left(v_{1}\right) v_{1}+2 \alpha_{\ddot{\xi}} \alpha_{\xi^{2}} x_{2} \cos x_{1}-m_{\xi} \alpha_{\xi} x_{2} \\
& \left.+\left(\alpha_{\ddot{\xi}} \alpha_{\xi}-m_{\xi} \alpha_{t}\right) \sin x_{1}-\alpha_{\ddot{\xi}} u\right] \\
= & -c_{1}\left(1+m_{\xi} g m \overline{G M}_{0}+m_{\xi} K_{\phi^{3}} x_{1}^{2}\right) x_{1}^{2} \\
& +\left[1-m_{\xi} g m \overline{G M}_{t} \cos \omega_{e} t\right] x_{1} \nu_{1}-c_{2} \nu_{1}^{2}
\end{aligned}
$$

with $u\left(x_{1}, v_{1}\right)$ given by

$$
\begin{aligned}
\alpha_{\ddot{\xi}} u \triangleq & |M| c_{1} v_{1}+2 \alpha_{\ddot{\xi}} \alpha_{\xi^{2}} x_{2} \cos x_{1}-m_{\xi} \alpha_{\xi} x_{2} \\
& +\left(\alpha_{\ddot{\xi}} \alpha_{\xi}-m_{\xi} \alpha_{t}\right) \sin x_{1}+c_{2} \nu_{1} \\
& -m_{\xi} \bar{K}_{\dot{\phi}}\left(v_{1}\right) v_{1}+\alpha_{\ddot{\xi}} d_{\xi}\left(v_{2}\right) v_{2} .
\end{aligned}
$$

$\dot{V}_{2}$ is negative definite for sufficiently large $c_{1}, c_{2}$.

As per the control objective, the other states must be at least bounded. The full closed-loop system reads

$$
\begin{aligned}
\dot{x}_{2}= & v_{2} \\
|M| \dot{v}_{2}= & -\frac{|M|}{\alpha_{\ddot{\xi}}} \alpha_{\xi} x_{2}+f_{t}\left(x_{1}, \nu_{1}\right) \\
\dot{x}_{1}= & -c_{1} x_{1}+\nu_{1} \\
|M| \dot{\nu}_{1}= & -c_{2} \nu_{1}-m_{\xi} g m \overline{G M}_{t} \cos \left(\omega_{e} t\right) x_{1} \\
& -m_{\xi} K_{\phi^{3}} x_{1}^{3}
\end{aligned}
$$

where

$$
\begin{aligned}
I_{\phi} \triangleq & I_{x x}-K_{\ddot{\phi}}+I_{t} \\
f_{t}\left(x_{1}, \nu_{1}\right)= & -\frac{|M|}{\alpha_{\ddot{\xi}}} \alpha_{t} \sin x_{1}+\frac{I_{\phi}}{\alpha_{\ddot{\xi}}}|M| c_{1} v_{1}+\frac{I_{\phi}}{\alpha_{\ddot{\xi}}} c_{2} \nu_{1} \\
& +\alpha_{\ddot{\xi}} K_{\phi^{3}} x_{1}^{3}-\frac{|M|}{\alpha_{\ddot{\xi}}} \bar{K}_{\dot{\phi}}\left(v_{1}\right) v_{1} \\
& +\alpha_{\ddot{\xi}} g m \overline{G M}(t) x_{1} .
\end{aligned}
$$


We note that the subsystem (16)-(17) is not dependent upon the system (14)-(15). With $V_{2}$ as the Lyapunov function, the origin of (16)-(17) is therefore globally exponentially stable by [12, Theorem 4.10].

The question then becomes whether or not the internal dynamics (14)-(15) are stable.

We note that $f_{t}$ is zero in zero, and that the zero-state dynamics of the $x_{2}-v_{2}$ subsystem is a pure, undamped oscillation.

We take $\bar{f}_{t}(t) \triangleq f_{t}\left(x_{1}(t), \nu_{1}(t)\right)$ to be an (analytically unknown) time-varying input function to the system (14)(15). Knowing that $x_{1}$ and $\nu_{1}$ go exponentially to zero and from the expression for $f_{t}$ we see that $\bar{f}_{t}$ is bounded by a decreasing exponential: $\left|\bar{f}_{t}(t)\right| \leq \gamma_{f} e^{-k_{f} t}$ for some $\gamma_{f}, k_{f}$.

Defining $\bar{\chi}_{2} \triangleq\left[x_{2}, v_{2}\right]$ and

$$
\Phi(t) \triangleq\left[\begin{array}{cc}
\cos \sqrt{\frac{\alpha_{\xi}}{\alpha_{\ddot{\xi}}}} t & \sin \sqrt{\frac{\alpha_{\xi}}{\alpha_{\ddot{\xi}}}} t \\
-\sin \sqrt{\frac{\alpha_{\xi}}{\alpha_{\ddot{\xi}}}} t & \cos \sqrt{\frac{\alpha_{\xi}}{\alpha_{\ddot{\xi}}}} t
\end{array}\right]
$$

the explicit solution to (14)-(15) can be found as

$$
\bar{\chi}_{2}(t)=\Phi(t) \bar{\chi}_{2}(0)+\int_{0}^{t} \Phi(t-\varsigma)\left[\begin{array}{c}
0 \\
\bar{f}_{t}(\varsigma)
\end{array}\right] \mathrm{d} \varsigma
$$

from which we can see that

$$
\begin{aligned}
\left\|\bar{\chi}_{2}(t)\right\| & \leq\|\Phi(t)\|\left\|\bar{\chi}_{2}(0)\right\|+\int_{0}^{t}\|\Phi(t-\varsigma)\|\left|\bar{f}_{t}(\varsigma)\right| \mathrm{d} \varsigma \\
& \leq\left\|\bar{\chi}_{2}(0)\right\|+\gamma_{f} \int_{0}^{\infty} e^{-k_{f} \varsigma} \mathrm{d} \varsigma=\left\|\bar{\chi}_{2}(0)\right\|+\gamma_{f}
\end{aligned}
$$

since $\|\Phi(t)\| \equiv 1$ (using the induced 2-norm). Thus we conclude that the $x_{2}-v_{2}$ subsystem of (14)-(15) is bounded.

Therefore, the control law of (13) will globally exponentially stabilize $\phi=0$, while keeping all the other states at least bounded, as per the control objective. The rolling dies out, while the motion of the tank fluid does not.

This effect might seem counter-intuitive; the fluid in the tank is moving from one reservoir to the other with no effect on the rolling motion. This is explained by the nature of the dynamic tank-induced roll moment $f_{\xi}(\xi, \ddot{\xi})=-\alpha_{\ddot{\xi}} \ddot{\xi}-\alpha_{\xi} \xi$. Setting the tank-generated moment to zero (i.e. when the tank has no effect on roll) gives the linear ODE $\alpha_{\ddot{\xi}} \ddot{\xi}+\alpha_{\xi} \xi=0$ whose solution is a standing sinusoid.

Using the tank-generates moment $f_{\xi}(\xi, \ddot{\xi})$ as the input into the roll equation and setting it to any control law that drives roll to zero (e.g. the controller designed in this paper) will, as the end result, leave the fluid in the tank oscillating as a standing sinusoid due to the nature of $f_{\xi}(\xi, \ddot{\xi})$.

This unwanted behavior could be countered with a more advanced controller. This remains future work.

\section{Simulations}

Simulation results can be seen in Figs. $3-5 . \phi(0)=5^{\circ}$, while the other variables started at zero. Some simulation parameters were

$$
\begin{aligned}
& c_{1}=21 / \mathrm{s} \\
& c_{2}=5 \mathrm{~kg}^{2} \mathrm{~m} \\
& A_{r}=30 \mathrm{~m}^{2} \\
& A_{d}=15 \mathrm{~m}^{2} \\
& \rho_{t}=1000 \mathrm{~kg} / \mathrm{m}^{3} \\
& w=27 \mathrm{~m} \\
& h_{r}=4 \mathrm{~m} \\
& r_{d}=2 \mathrm{~m} \\
& q_{v}=5 \mathrm{~m}^{2} / \mathrm{s} \\
& q_{|v| v}=3 \mathrm{~m}
\end{aligned}
$$

The other simulation parameters can be found in [9, exp. 1174]. Total maximum input is limited to $|u| \leq \pm 6 \mathrm{MN}$, and water level difference limited to $\xi \leq \pm 2 h_{r}= \pm 8 \mathrm{~m}$.

Simulation results for the controlled nominal system can be seen in Fig. 3. The controller is able to drive the roll angle $\phi$ rapidly to zero, while the tank level $\xi$ becomes a standing sinusoid, in accordance with theoretical results.

Simulation results for the controlled plant model with the controller designed for the nominal system can be seen in Fig. 4. The controller is able to drive the roll angle $\phi$ rapidly to zero, while the tank level $\xi$ becomes a standing sinusoid.

The overshoot in Figs. 3 and 4 is due to limiting $u$ and $\xi$. Without saturation, $\phi(t) \approx \phi(0) e^{-c_{1} t}$, but $\xi$ reaches unphysical values.

For comparison, the plant was also simulated with only a passive tank, and without a tank at all (Fig. 5).

\section{CONClusion}

In this paper, we have considered a model of ship parametric roll resonance and an active u-tank stabilizer. We have designed a controlled using Lyapunov theory that achieves global exponential stability for roll motion. The performance of the controller has been tested with a high-fidelity simulation model, and the results are very encouraging. In the presented simulations, the water level in the tank remains oscillating once the roll motion has subsided. This unwanted effect can be negated, but this goes beyond the scope of this paper.

\section{REFERENCES}

[1] A. H. Nayfeh and D. T. Mook, Nonlinear Oscillation. Wiley VCH, 1995.

[2] W. N. France, M. Levadou, T. W. Treakle, J. R. Paulling, R. K. Michel, and C. Moore, "An investigation of head-sea parametric rolling and its influence on container lashing systems," in SNAME Annual Meeting, 2001.

[3] S. M. Carmel, "Study of parametric rolling event on a panamax container vessel," Journal of the Transportation Research Board, vol. 1963, pp. 56-63, 2006.

[4] R. Galeazzi, C. Holden, M. Blanke, and T. I. Fossen, "Stabilization of parametric roll resonance by combined speed and fin stabilizer control," submitted to the ECC09.

[5] E. V. Lewis, Ed., Principles of Naval Architecture. The Society of Naval Architects \& Marine Engineers, 1988.

[6] M. A. S. Neves, J. Merino, C. Rodríguez, and L. Soares, "On the use of u-type stabilizing tanks for reducing roll motions in head seas," International Shipbuilding Progress, vol. 51, no. 2, pp. 221-235, 2004.

[7] A. R. J. M. Lloyd, Seakeeping: Ship Behaviour in Rough Weather. A R J M Lloyd, Gosport, United Kingdom, 1998.

[8] R. Moaleji and A. R. Greig, "On the development of ship anti-roll tanks," Ocean Engineering, vol. 34, no. 1, pp. 103-121, 2007.

[9] C. Holden, R. Galeazzi, C. Rodríguez, T. Perez, T. I. Fossen, and M. A. S. Neves, "Nonlinear container ship model for the study of parametric roll resonance," Modeling, Identification and Control, vol. 28 , no. 4, pp. 87-103, 2007.

[10] R. Galeazzi and M. Blanke, "On the feasability of stabilizing parametric roll with active bifurcation control," in Proceedings of the IFAC Conference on Control Applications in Marine Systems, 2007.

[11] T. I. Fossen, Marine Control Systems: Guidance, Navigation and Control of Ships, Rigs and Underwater Vehicles. Marine Cybernetics, 2002.

[12] H. K. Khalil, Nonlinear Systems, 3rd ed. Prentice Hall, 2000. 


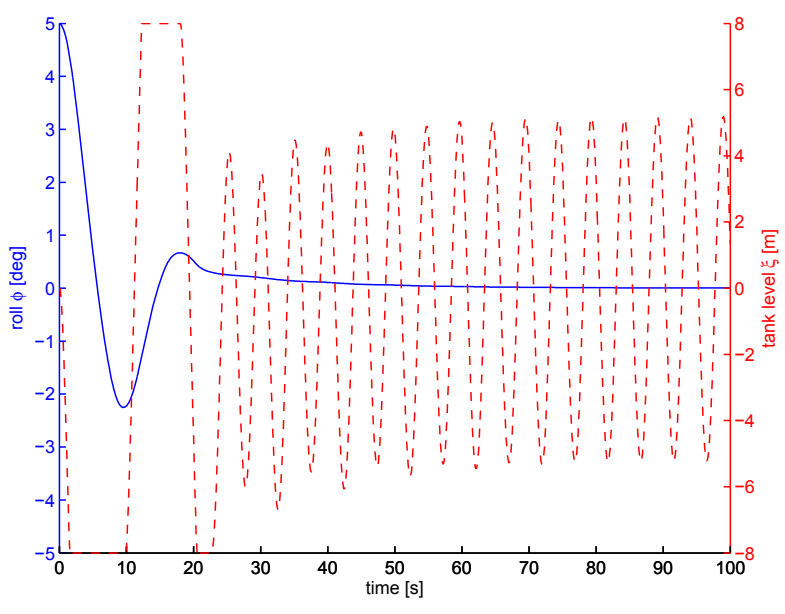

(a) Roll angle $\phi$ (solid) and tank level $\xi$ (dashed).

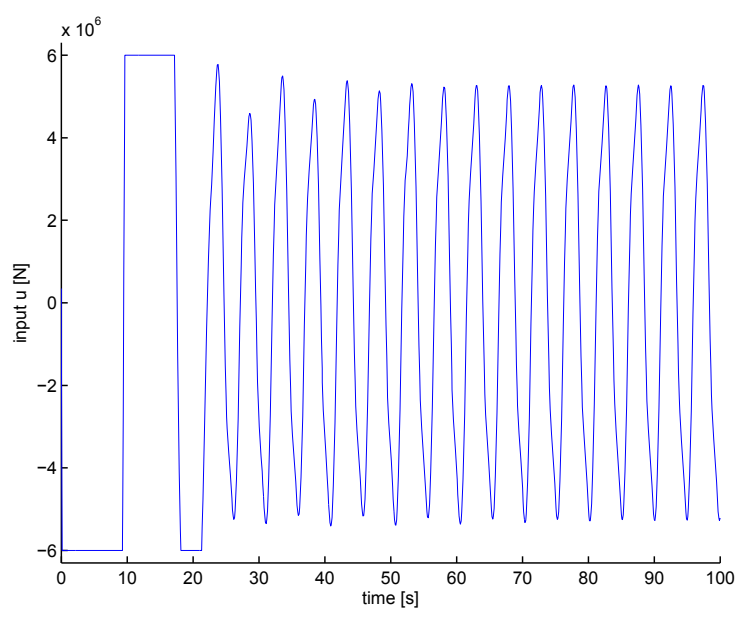

(b) Control input $u$.

Fig. 3. Simulation results for the controlled nominal model (11)-(12).

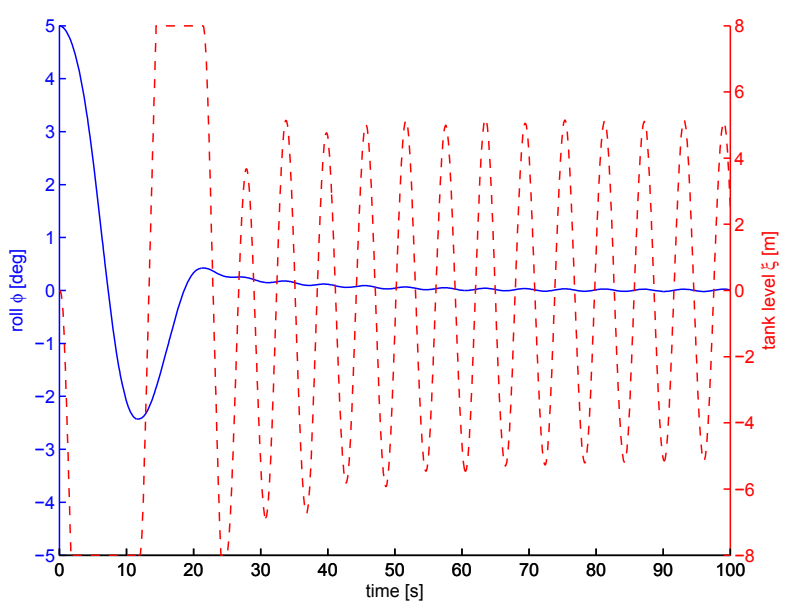

(a) Roll angle $\phi$ (solid) and tank level $\xi$ (dashed).

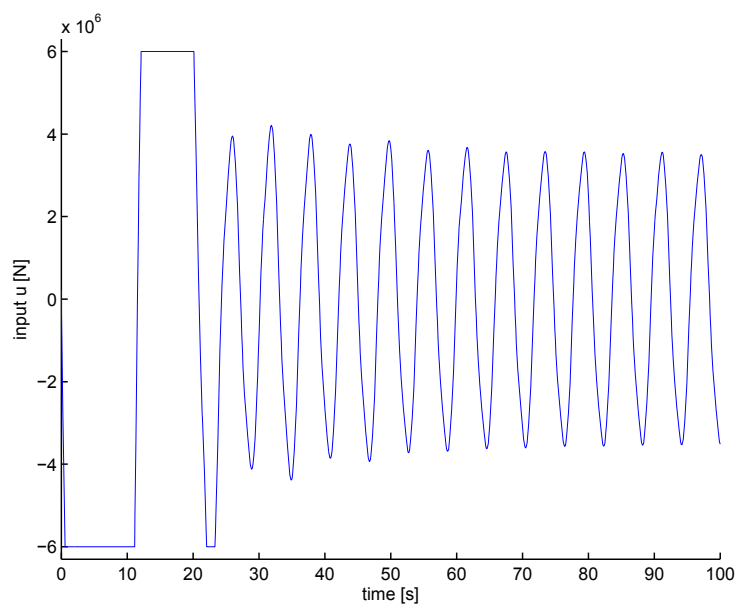

(b) Control input $u$.

Fig. 4. Simulation results for the controlled plant model (10).

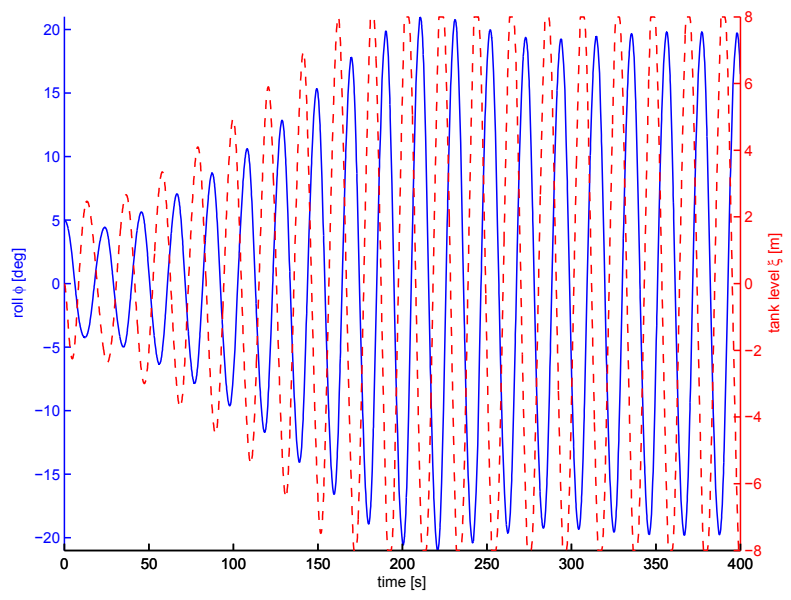

(a) Passive tank. Roll angle $\phi$ (solid) and tank level $\xi$ (dashed).

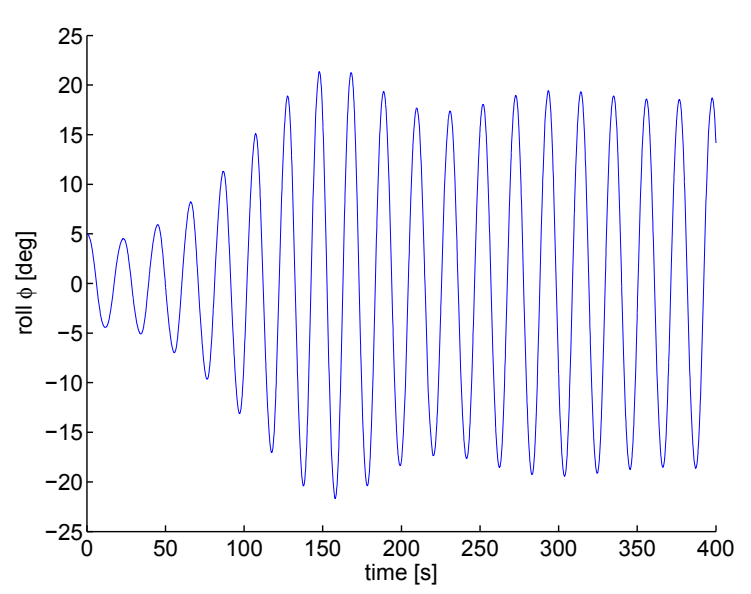

(b) No tank.

Fig. 5. Simulation results for the uncontrolled plant model. 